\title{
Registration Technology Based on Image Feature
}

\author{
Wang Fang \\ East China Institute of Technology
}

\author{
Wang Xiaoyan \\ East China Institute of Technology
}

\begin{abstract}
Image Registration is that to match among two or more than two images with the same target in spatial position. Registration Technology based on image feature has two important sections including feature extraction and feature matching. We ca choose feature including points, lines and areas. This paper focuses on implementation procedure of Image Registration Technology based on image feature.
\end{abstract}

Keywords-Registration, feature points, transformation, matching

\section{INTRODUCTION}

Registration Technology based on image feature is to make use of some feature to match images. Select some pixels of feature, extract feature including characteristic points, object edge, topography characteristic lines such as spinal lines, rivers, roads, angles and so on, and then utilize these feature to calculate spatial transformation parameters. According to different extracted feature, feature extraction operator has point feature operator, line feature operator and plane feature operator. The base steps and methods are coincidental such as Figure 1.

\section{REGISTRATION STEPS}

\section{A. Image Processing}

It is to eliminate or reduce gradation deviation and geometry deformation among images to be registered. And it will make the matching process go on smoothly.

\section{B. feature selecting}

It is to select intersection of boundary lines, obvious feature of region outline in images or to utilize feature to extract the operator.

\section{C. feature matching}

It is to realize image matching on corresponding obvious feature points between the two images and to regard these matched feature points as control points or common points. We must note the following aspects when we select the control points, one is to distribute as even as possible, two is to have obvious recognition mark in the corresponding image, three is to guarantee a certain number of points.

\section{Spatial Transformation}

It is to match $\mathrm{I} 1(\mathrm{x}, \mathrm{y})$ and $\mathrm{I} 2(\mathrm{x}, \mathrm{y})$ between the two images from different time or different device. That is to find a mapping relation $\mathrm{P}:(\mathrm{x} 1, \mathrm{y} 1) \rightarrow(\mathrm{x} 2, \mathrm{y} 2)$, make every point of I1 correspond with an unique point of $\mathrm{I} 2$, and make sure that the two points correspond the same anatomical position. $\mathrm{P}$ is regarded as a group of contiguous spatial transformation. The common spatial transformations are Rigid Body
Transformation, Affine Transformation, Projective Transformation and Nonlinear Transformation.

\section{1) Rigid Body Transformation}

If the distance between any two points in the first image is unchanged after transformation into the second image. For example, head is regarded as a rigid body. Registration of head images always uses Rigid Body Transformation. It can be broken down into rotation and translation:

$$
p(x)=a x+b
$$

$x=(x, y, z)$ is the pixel's spatial location, $a$ is a $2 \times 2$ rotation matrix, $b$ is a $2 \times 1$ translation vector. The matrix should satisfy the constraints::

$$
\begin{aligned}
& a^{T} a=I \quad \operatorname{det} I=1 \\
& a^{T} \text { is transpose of a matrix } a, I \text { is unit matrix. }
\end{aligned}
$$

\section{2) Affine Transformation}

If the constraints of (2) are dissatisfied, then the equation of (1) will describe Affine Transformation. It will map the line to line and maintain parallelism. The specific performance can be uniform scale transformation with identical scales of every direction or non-uniform scale transformation or cut transformation with inconsistent scales. Uniform scale transformation is more used in photographic images in lens system. In this case, there is direct relation between image and imaging optics. Generally this transformation can be used in correcting distortion generated by cut or gradient coils because of tilted CT bench.

\section{3 ) Projective Transformation}

It is similar to Affine Transformation. It will map the line to line but not maintain parallelism. It is mainly used in registration of two-dimensional projection images and three-dimensional volume images.

\section{4) Nonlinear Transformation}

It is also named Curved Transformation and it change line to curve. It more uses polynomial function, such as quadratic function, cubic function, plate spline function and an exponential function. It is always used in registration of images fitted by Anatomical atlas deformation or Chest, abdominal organs images with global deformation.

\section{E. Resampling}

When we transform the images, we need to rearrange the pixels according to the transformation relation. The transformation always needs Resampling technology which is regarded as interpolation to approximately calculate gray value of pixel after coordinate transformation. The common interpolation methods are nearest neighbor interpolation, bilinear interpolation and high-degree interpolation. In this 
step is to reassign the gray value of images to be registered by gray transformation.

\section{1) Nearest neighbor interpolation}

It is a simple interpolation method, and it is also named zero-order interpolation. Its output pixel gray value is equal to nearest input pixel gray value. This method is simple and intuitive, but it will generate processing traces while pixels including fine structure with gray changes. The formula is that:

$$
\begin{gathered}
f(x, y)=f([x+0.5],[y+0.5]) \\
\text { [ ]is a rounding symbol. }
\end{gathered}
$$

\section{2) Bilinear interpolation}

It is also called first-order interpolation, and it is better than nearest neighbor interpolation. The calculation is very great but the image quality after interpolation is so high that there is not discontinuous pixel. Furthermore, because it has the nature of low-pass filter and damage to high-frequency components, so it may make image profile blurred to some extent. The formula is that:

$$
\begin{gathered}
f(x, y)=f(i, j)(1-\alpha)(1-\beta)+f(i+1, j) \alpha(1-\beta) \\
+f(i, j+1)(1-\alpha)(1-\beta)+f(i+1, j+1) \alpha \beta \\
i=[x], \quad y=[j], \quad \alpha=x-i, \quad \beta=y-j
\end{gathered}
$$

\section{3) High-order interpolation}

High-order interpolation commonly use cubic convolution. It can conquer the shortcomings of the two algorithms ahead, calculate with high precision, but the calculation is too great. it considers 16 consecutive points around a floating point coordinate.

$$
f(x, y)=[A][B][C]
$$

In this formula,

$$
\begin{aligned}
& {[\mathrm{A}]=\left(\begin{array}{llll}
S(1+u) & S(u) & S(1-u) \quad S(2-u)
\end{array}\right),[\mathrm{C}]=\left(\begin{array}{c}
S(1+v) \\
S(v) \\
S(1-v) \\
S(2-v)
\end{array}\right)} \\
& {[B]=\left(\begin{array}{cccc}
f(i-1, j-1) & f(i-1, j) & f(i-1, j+1) & f(i-1, j+2) \\
f(i, j-1) & f(i, j) & f(i, j+1) & f(i, j+2) \\
f(i+1, j-1) & f(i+1, j) & f(i+1, j+1) & f(i+1, j+2) \\
f(i+2, j-1) & f(i+2, j) & f(i+2, j+1) & f(i+2, j+2)
\end{array}\right)} \\
& \mathrm{S}(w)=\left\{\begin{array}{c}
1-2|w|^{2}+|w|^{3},|w|<1 \\
4-8|w|+5|w|^{2}-|w|^{3}, 1<=|w|<2 \\
0,|w|>=2
\end{array}\right. \\
& \text { It is close to } \mathrm{S}(w)=\frac{\sin (w)}{w}
\end{aligned}
$$

Generally the image transformation is controlled by parameters. The coordinate relationship is got by $\mathrm{I} / \mathrm{O}$ variable formula and parameters. If the image coordinate is $(x, y)$, then it change to $(u, v)$, the transformation relationship usually is that:

$$
(u, v)=\left(f x(x, y), f y(x, y),(u, v) \in R^{2},(x, y) \in I^{2}\right.
$$

So that, the coordinate after transformation is real coordinate. This transformation is called forward mapping transformation. For digital images, the image pixel after transformation needs to arrange in order in the integer coordinate location, so that if use the forward mapping transformation, there will appear holes and aliasing. In actual processing, in order to get the continuous images, to suppose the integer coordinate is $(u, v)$, and to get the real coordinate $(x, y)_{\text {by inverse transformation in the reference }}$ images.

$$
(x, y)=(f u(u, v), f v(u, v)),(x, y) \in R^{2},(u, v) \in I^{2}
$$

The pixel of $(x, y)$ can be calculated by $\mathrm{n}$ arest neighbor interpolation in the reference images. This transformation relationship between output integer coordinate and input coordinate is called inverse mapping. Image Resampling always use inverse transformation to get the image coordinate transformation.

In order to obtain better picture results and to prevent unnatural phenomenon, Karl.M offers a real-time antialiasing space transformation technology. It amends pixel gray value in the integer coordinate point by point after transformation, then it realizes the fast continuous Resampling, so it can eliminate holes and aliasing. With gray transformation, the gray value of preparing images after space transformation will be revalued.

Registration Technology based on image feature is applied in fact more and more extensive. Image feature just care partial information, so that it reduces amount of calculations to increase the speed of registration. At the same time, the matching measure of feature points is very sensitive to position and that can improve the matching precision. However, this method only depends on image feature, so it is not very sensitive to minor details of images.

In Figure 2, the left one is image to be registered, and the right one is reference image.

Figure 4 shows the registering results using the method of selecting image feature points.

\section{SUMMARIES}

Of course, in actual process of image Registration we need to select appropriate method according to actual situation and needs of registration. And we also can combine several methods to use. If we use the faster method to make the initial registration and then use the more complex method to make the father registration, so that we can achieve better registration results.

\section{REFERENCES}

[1] Gong Shengrong,Liu,Chunpin,Wangqiang.Digital image processing and analysis[M].Tsinghua University Press,2006.

[2] He Dongjian, Geng Nan.Digital image processing[M].Xidian University Press, 2003.

[3] Ma Dong,Sun Yongxing,Cheng Jingzhi.Multi-Modal fusion and registration of medical image[J].Journal of Biomedical Engineering, 1999 .

[4] Gunilla Borgefors,Hierarchical Chamfer Matching.A Parametric Edge Matching Algorithm[J]. IEEE TRANSACTIONS ON PATTERN ANALYSIS AND INTELLIGENCE.VOL.10.NO 6.NOVEMBER 1988: 849-865.

[5] Hui Li,B . S Manjuanth,Sanjit K, Mitra.A Contour-Based Approach to Multisensor Image Registration[J].IEEE TRANSACTIONS ON IMAGE PROCESSING.VOL 4.NO 3,MARCH 1995:320-334. 
[6] Derek L G Hill,Philipp G Batchelor,Mark Holden and David J Hawkes.Medical Image Registration[J]. PHYSICS IN MEDICINE AND BIOLOGY.VOL 46,2001: 1-45.

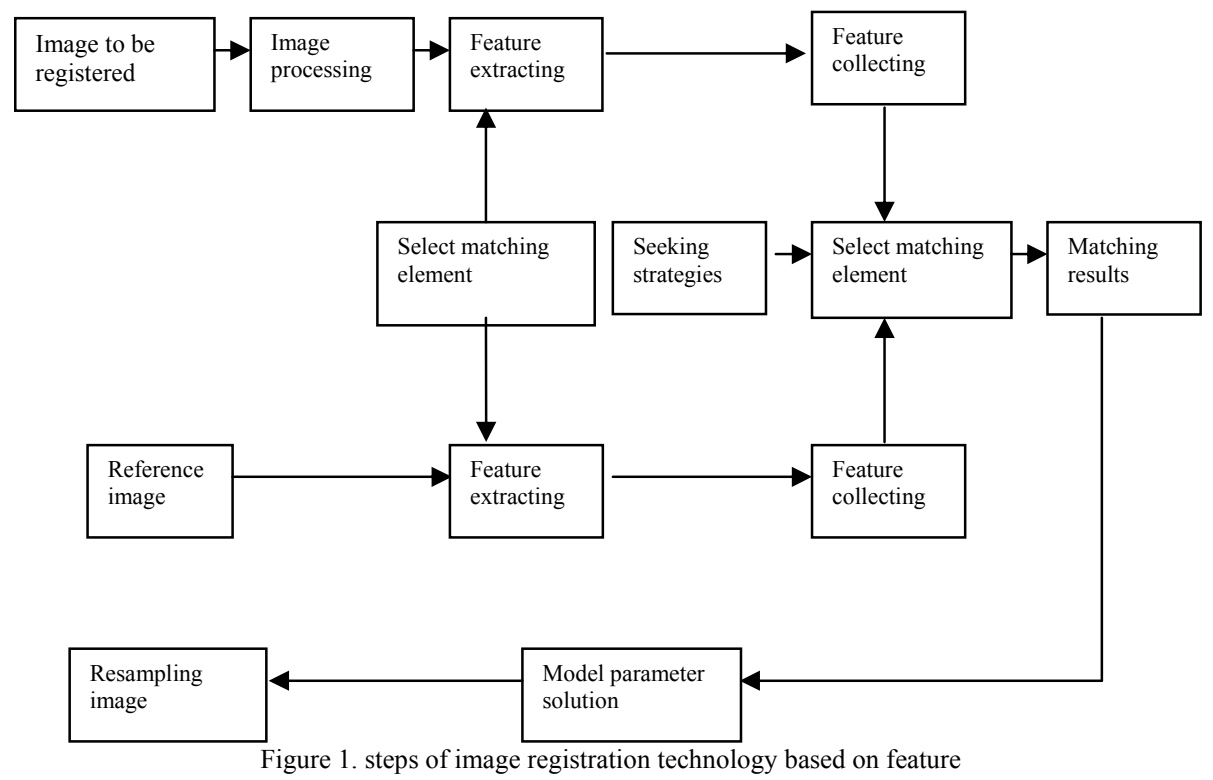

Figure 1. steps of image registration technology based on feature

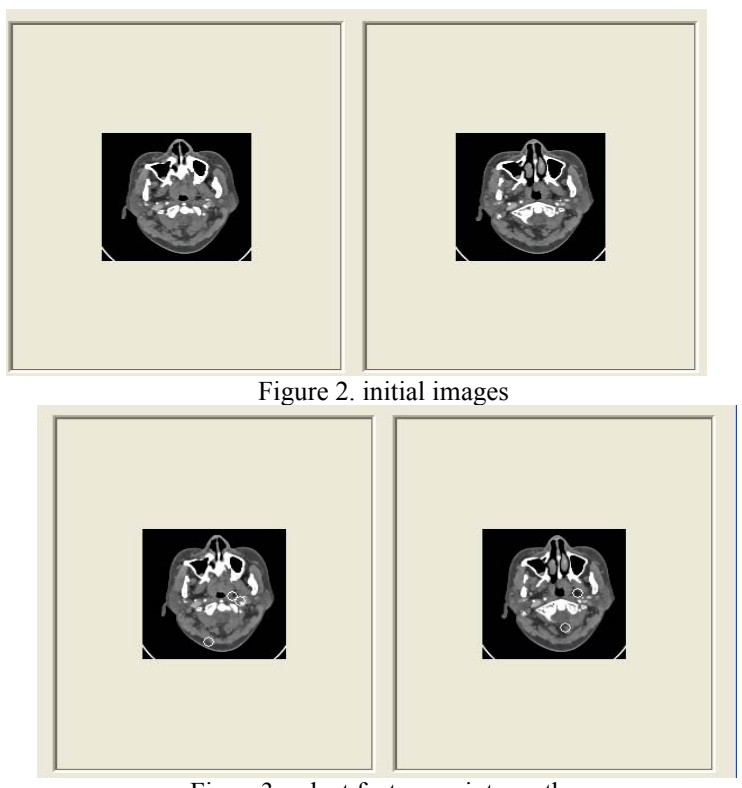

Figure3. select feature points on the two images

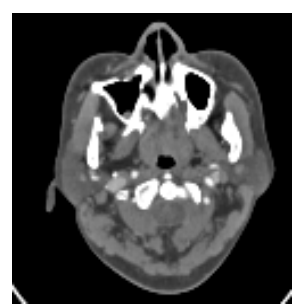

Figure 4. image registration based on feature point 